\title{
Seguridad alimentaria, crecimiento y niveles de vitamina A, hemoglobina y zinc en niños preescolares del nordeste de Brasil
}

\author{
Food security, growth and vitamin A, hemoglobin \\ and zinc levels of preschool children in the northeast of Brazil
}

Dixis Figueroa Pedraza ${ }^{1}$

Daiane de Queiroz ${ }^{2}$

Adriana de Azevedo Paiva ${ }^{3}$

Maria Auxiliadora Lins da Cunha ${ }^{4}$

Zilka Nanes Lima ${ }^{4}$

${ }^{1}$ Programa de Pós-

Graduação em Saúde

Pública, Universidade

Estadual da Paraíba. Av. das

Baraúnas 351 Campus

Universitário, Bairro

Bodocongó. 58.109-753

Campina Grande Paraíba

Brasil.

dixisfigueroa@gmail.com

${ }^{2}$ Faculdade de Ciências

Médicas de Campina

Grande.

${ }^{3}$ Universidade Federal do

Piauí.

${ }^{4}$ Universidade Estadual da

Paraíba.
Abstract This study sought to examine the association between the food (in) security and nutritional status of preschool children attended in daycare centers. Food security was assessed using the Brazilian Food Insecurity Scale. The nutritional status was evaluated using the weight/ height, weight/age, height/age, hemoglobin, serum retinol and serum zinc status. The prevalence of stunting (6.2\%), overweight (3.1\%), underweight (2.1\%), vitamin A deficiency (24.4\%), anemia (15.5\%), and zinc deficiency (15\%) was established. Food insecurity was found in $64.4 \%$ of the families, predominantly in its mild form (32.6\%). This study concludes that food insecurity as measured by the EBIA was not associated with $Z$-score growth or with vitamin $A$, hemoglobin and zinc biochemical concentrations. Key words Food insecurity, Hemoglobin, Serum retinol, Serum zinc, Preschool
Resumen El presente estudio buscó analizar la asociación entre la (in)seguridad alimentaria y el estado nutricional de niños preescolares asistidos en jardines infantiles. La seguridad alimentaria fue evaluada a través de la Escala Brasileña de Inseguridad Alimentaria (EBIA). El estado nutricional se evaluó a través del peso/edad, tallal edad, hemoglobina, retinol sérico y zinc sérico. Fueron encontradas prevalencias de déficit de estatura (6,2\%), déficit de peso/edad (2,1\%), deficiencia de vitamina A (24,4\%), anemia (15,5\%) $y$ deficiencia de zinc (15,0\%). La inseguridad alimentaria familiar fue caracterizada en $64,2 \%$ de las familias predominando la forma leve (32,6\%). El estudio concluye que la inseguridad alimentaria estimada por la EBIA no se asoció a los Escore$z$ de crecimiento ni a las concentraciones de retinol sérico, hemoglobina y zinc sérico.

Palabras clave Inseguridad alimentaria, Hemoglobina, Retinol sérico, Zinc sérico, Preescolar 


\section{Introducción}

La Seguridad Alimentaria y Nutricional (SAN) puede y debe ser comprendida como la condición que asegura a todas las personas, en todos los espacios geográficos, en todo momento, acceso físico, económico y cultural a un elenco básico de alimentos capaz de suplir, de forma regular y permanente, sus necesidades biológicas de energía y nutrientes, segundo las recomendaciones de los comités internacionales habilitados por las Naciones Unidas para tratar del asunto. Esta condición debe ser asegurada en un contexto de plena satisfacción a otros derechos fundamentales, como educación, vivienda, salud y bienestar social, dentro de un proceso autónomo y sustentable de desarrollo, teniendo como soporte el ejercicio de una actividad laboral ética y culturalmente aceptable. Así, la SAN se concretiza, en términos de salud, con el bienestar nutricional ${ }^{1,2}$.

En ese contexto la SAN debe ser medida, en términos poblacionales, en grupos específicos, en instancia familiar e individual, con uno o varios instrumentos considerando parámetros de precisión, confiabilidad y utilidad. La escala propuesta por Radimer et al. ${ }^{3}$ viene siendo validada y aplicada en varios países de América Latina para dimensionar la magnitud de la inseguridad alimentaria $^{4-6}$. Tomando como referencia el enfoque propuesto por Karl Radimer en su tesis doctoral y otras escalas similares, como la del Community Childhood Hunger Identification Project (CCHIP), estudiosos del Departamento de Agricultura de los Estados Unidos perfeccionaron el método y posteriormente fue adoptado como instrumento básico en las investigaciones de salud y nutrición de los Estados Unidos ${ }^{1,7}$. En Brasil, la escala fue adaptada y validada, resultando en la Escala Brasileña de Inseguridad Alimentaria (EBIA) $)^{8}$, único instrumento validado para la realidad brasileña idóneo para evaluar de forma directa la (in)seguridad alimentaria9.

La EBIA viene demostrando su aplicación de manera satisfactoria, siendo pertinente considerar que Brasil es el país que reúne la experiencia más extensa del mundo en términos de uso masivo de ese tipo de instrumento ${ }^{1}$. Su aplicación ha resultado en reflexiones importantes al considerarlo como instrumento de evaluación: i) fundamento adecuado en la percepción relacionada a la preocupación sobre los problemas relativos a la cantidad y calidad de la alimentación fundamenta, ii) posibilita de forma práctica y rápida el diagnóstico directo de la situación de seguridad alimentaria familiar, iii) indicador sensible para detectar familias en riesgo de inseguridad alimen$\operatorname{taria}^{1,10,11}$.

En 1990, la Reunión de Cúpula de Nueva York $^{12}$ apud Oliveira et al. ${ }^{1}$, expresó como parte de la estrategia para mejorar el estado nutricional de niños y mujeres de países en desarrollo que la SAN, además de consenso y objetivo en sí, constituye un prerrequisito para prevenir deficiencias nutricionales, como el bajo peso al nacer, la desnutrición energético-proteica, la hipovitaminosis A, las anemias y la deficiencia de yodo. En esas circunstancias, consideramos oportuno analizar la relación entre los niveles de (in)seguridad alimentaria y el estado nutricional basado en indicadores antropométricos y marcadores bioquímicos de carencias de micronutrientes. Entretanto, son escasas las publicaciones relacionando indicadores directos de (in)seguridad alimentaria, como la EBIA, e indicadores del estado nutricional de niños. Algunos trabajos han sido desarrollados para evaluar la asociación entre la (in)seguridad alimentaria y la desnutrición en niños, mostrando resultados diversos ${ }^{9,13-15}$. Sin embargo, la asociación de la (in)seguridad alimentaria con deficiencias de micronutrientes fue considerada en un único estudio ${ }^{16}$.

Considerando que: i) uno de los objetivos de la SAN debe ser el bienestar nutricional ${ }^{1}$, ii) altas prevalencias de inseguridad alimentaria han sido registradas en diferentes estudios brasileños ${ }^{9-11}$, iii) el contexto brasileño actual de menores prevalencias de desnutrición infantil y mayores de deficiencias de micronutrientes ${ }^{17}$, con cuadro epidemiológico que se asemeja más al de la seguridad alimentaria, tanto en términos de frecuencias como de alcance a grupos de menor vulnerabilidad; explicaciones para la relación entre los niveles de (in)seguridad alimentaria con el estado nutricional pueden estar basadas en indicadores directos del estado nutricional, como los marcadores bioquímicos de deficiencias de micronutrientes, con los cuales, tal vez, pueden presentar mayor linealidad, y no basadas únicamente en indicadores antropométricos.

De esa forma, el presente estudio tiene por objetivo analisar la asociación entre la (in)seguridad alimentaria y el estado nutricional de niños preescolares asistidos en jardines infantiles, considerando los Escore-z de crecimiento y las concentraciones de retinol sérico, hemoglobina y zinc sérico. Las prevalencias de las características de interés son determinadas de manera preliminar para caracterizar el perfil de vulnerabilidad nutricional de los individuos de estudio. Se espera que los resultados del presente estudio 
Estudio transversal cuyos datos son provenientes del proyecto "Crecimiento, desarrollo cognitivo y deficiencias de micronutrientes: perfil de los niños asistidos en el Núcleo de Jardines Infantiles del Gobierno de Paraíba”. El proyecto fue aprobado por el Comité de Ética de la Universidade Estadual da Paraíba. Fueron contempladas todas las normas que reglamentan las investigaciones con seres humanos - Resolución 196/96 ${ }^{18}$, del Conselho Nacional de Saúde do Brasil.

Fueron elegibles para el estudio niños preescolares frecuentadores de jardines infantiles públicos del gobierno del Estado de Paraíba, Brasil. La definición del grupo poblacional se basó en la posibilidad de estudiar familias de vulnerabilidad socio-económica y con núcleo constituido por niños pre-escolares. En este estudio se excluyeron los niños mayores de 60 meses.

En total funcionan 45 jardines infantiles en barrios distintos de las ciudades beneficiadas, situados, generalmente, en áreas carentes. El beneficio está presente en ocho municipios del Estado de Paraíba: João Pessoa (30 jardines infantiles), Campina Grande ( 9 jardines infantiles), además de las ciudades de Areia, Bayeux, Mamanguape, Itaporanga, Soledade y Umbuzeiro (cada una de ellas con un jardín infantil). El universo es de 3310 niños beneficiados, distribuidos, aproximadamente, en 2317 en el municipio de João Pessoa, 621 en el municipio de Campina Grande y 372 en los otros municipios.

La opción para determinar el tamaño de la muestra de estudio fue el procedimiento de muestreo para proporciones: donde $\mathrm{N}$ es el total de la población, $Z \alpha^{2}=1.96^{2}$ ( si la confianza es de 95\%), p es la proporción esperada, $q=1-p$, d es la precisión arbitraria (error de estimación). Se consideró $\mathrm{p}=7,0 \%$ (media del déficit de talla en Bra$\mathrm{sil}^{19}$ ) y $\mathrm{d}=3 \%$, totalizando 256 individuos. Ese valor fue corregido en $10 \%$ para compensar eventuales pérdidas, estableciéndose una muestra de 282 niños.

Fue seleccionada una muestra probabilística de jardines infantiles e individuos, utilizando procedimiento en dos etapas. Para garantizar la representatividad de los municipios, el sistema de referencia para la primera etapa fue ordenado

según estratos (João Pessoa, Campina Grande, otros municipios), posibilitando la obtención de tamaños de muestras apropiados para cada estrato. Así, se definió la necesidad de estudiar 14 jardines infantiles para completar la muestra, 11 en João Pessoa, dos en Campina Grande e un en otros municipios. En la segunda etapa, fueron sorteadas los niños de forma aleatoria en el momento del trabajo de campo.

Todos los procedimientos fueron realizados por equipe entrenada. Fue realizada dupla digitación de los datos en planillas del programa Excel (Microsoft Inc., Estados Unidos), de manera tal que posibilitó la unificación entre los mismos, a través de una única variable de identificación. Terminado el proceso de digitación, los dos bancos de datos fueron cruzados con la utilización del aplicativo Validate del programa Epi Info v. 6.04b (WHO/CDC, Atlanta, Estados Unidos), posibilitando verificar errores de digitación y generar el banco final que fue usado para las análisis estadísticas.

\section{Evaluación de la (in)seguridad alimentaria}

Las informaciones sobre (in)seguridad alimentaria fueron prestadas por los responsables de los niños, en su mayoría las madres, encargadas por la alimentación de la familia. Para su evaluación se utilizó la EBIA. Este instrumento consta de 15 preguntas centrales cerradas referentes a la experiencia de insuficiencia alimentaria en los últimos tres meses y diversos niveles de intensidad, que va de la aprensión relacionada a la posibilidad de la comida faltar hasta la vivencia de pasar un día completo sin comer, pudiendo afectar todos o algún miembro de la familia. De las 15 preguntas, seis se refieren específicamente a los niños y/o adolescentes ${ }^{20,21}$.

Los niveles de seguridad alimentaria familiar fueron establecidos por medio de la cuantificación del total de respuestas afirmativas en la escala:

a) Seguridad Alimentaria: 0 respuesta positiva

b) Inseguridad Alimentaria Leve: 1-5 respuestas positivas

c) Inseguridad Alimentaria Moderada: 6-10 respuestas positivas

d) Inseguridad Alimentaria Grave: 11-15 respuestas positivas

\section{Evaluación del estado nutricional con la utilización de indicadores antropométricos}

La evaluación antropométrica de los niños fue realizada siguiendo los procedimientos técni- 
cos recomendados por la Organización Mundial de la Salud $(\mathrm{OMS})^{22}$. Fueron tomadas las medidas de peso $(\mathrm{g})$ y talla $(\mathrm{cm})$. El peso se obtuvo con auxilio de pesa electrónica (Tanita UM- $080^{\circledR}$ ) con capacidad para $150 \mathrm{~kg}$ y precisión de $100 \mathrm{~g}$. La talla de los niños menores de 24 meses se obtuvo con auxilio de infantómetro de madera (construcción propia) con amplitud de $130 \mathrm{~cm}$ y subdivisiones de $0,1 \mathrm{~cm}$. En los niños mayores de 24 meses se utilizó estadiómetro $\left(\mathrm{WCS}^{\circledR}\right.$ ) con amplitud de $200 \mathrm{~cm}$ y subdivisiones de $0,1 \mathrm{~cm}$. Para garantizar la precisión, las medidas fueron tomadas dos veces para cada individuo, siendo utilizada la media de cada medida para efecto de registro de la variable. La edad del niño fue calculada en meses, mediante la diferencia entre la fecha de nacimiento (obtenida del certificado de nacimiento o de la ficha de salud) y la fecha de la entrevista.

La clasificación del estado nutricional se realizó con base en los índices peso para la edad (P/E) y talla para la edad (T/E), expresos en Escore-z. Los cálculos fueron realizados con el programa WHO Anthro 2005 versión beta. Como padrón de referencia se utilizó la recomendación de la OMS (Multicentre Growth Reference Study) ${ }^{23}$, adoptando como categorías de diagnóstico nutricional el déficit de peso para la edad y el déficit de estatura sobre la base de los pontos de corte $\mathrm{P} / \mathrm{E}<-2$ Escore-z y T/E $<-2$ Escore-Z, respectivamente.

\section{Evaluación del estado nutricional con la utilización de marcadores bioquímicos de deficiencias de micronutrientes}

Las muestras de sangre se obtuvieron de una vena del antebrazo. Fueron colectados $6 \mathrm{~mL}$ de sangre por técnicos con experiencia en la colecta de sangre en niños. Las muestras de sangre fueron colectadas usando material descartable, el suero fue separado por centrifugación a 3.000 rpm, por un período de 10 a 15 minutos, y las muestras congeladas posteriormente. Tubos con anticoagulante $\mathrm{K}_{3}$ EDTA fueron usados para las muestras de hemoglobina, tubos transparentes "trace free" para las muestras de zinc sérico y tubos transparentes sin anticoagulante envueltos en papel aluminio, tapados inmediatamente, para las muestras de retinol sérico.

Los niveles séricos de retinol fueron determinados por el método de Cromatografía Líquida de Alta Eficiencia (HPLC), de acuerdo con la metodología descripta por Furr et al. ${ }^{24}$. Niños con concentraciones de retinol sérico $<0,7 \mu \mathrm{mol} /$ $\mathrm{L}$ fueron consideradas con deficiencia de vitami- na $\mathrm{A}^{25}$. Las determinaciones fueron realizadas en el Centro de Investigaciones en Micronutrientes de la Universidade Federal da Paraíba.

Para evaluar el estado nutricional de hierro fue utilizada la medida de hemoglobina a través de la realización de hemograma en contador automático (Sysmex SF - 3000, Roche Diagnóstica) conforme orientaciones del fabricante. Valores de hemoglobina $<110 \mathrm{~g} / \mathrm{L}$ fueron utilizados para la identificación de anemia ${ }^{26}$. Las determinaciones fueron realizadas en el Laboratorio de Análisis Clínicas de la Universidade Estadual da Paraíba.

Los niveles de zinc fueron determinados mediante Espectrofotometría de Absorción Atómica de Llama, empleando Espectrofotómetro Analyst 300 (Perkin-Elmer Norwalk, Ct, EUA) modelo 3100 a una longitud de onda de $213 \mathrm{~nm}$ y con aire-acetileno ${ }^{27}$. Concentraciones de zinc sérico $<65 \mu \mathrm{g} / \mathrm{dL}$ fueron consideradas para indicar su deficiencia ${ }^{28}$. Las determinaciones fueron realizadas en el Centro de Referencia Instituto Hermes Pardini.

La presencia de infección subclínica fue controlada a través de la determinación de PCR, por técnica Aglutinación de Látex (Human GMBH, Wiesbaden, Germany) según orientaciones del fabricante. Valores de PCR $\geq 6,0 \mathrm{mg} / \mathrm{L}$ fueron utilizados para la identificación de infección subclínica ${ }^{29}$. Las determinaciones fueron realizadas en el Laboratorio de Análisis Clínicas de la Universidade Estadual da Paraíba.

\section{Análisis estadísticas}

Para análisis de la consistencia interna de las variables, relacionadas a la EBIA, se utilizó el Alpha de Cronbach con un valor mínimo aceptable de 0,70 y valor máximo esperado de $0,90^{30}$.

Los análisis de asociación estadística de este estudio consideraron los niños que tuvieron valores de PCR adecuados y todos sus marcadores bioquímicos evaluados. Para testar posibles diferencias relacionadas al sexo, edad y seguridad alimentaria entre el grupo de niños analizados y el grupo de niños que no formaron parte de los análisis, fueron aplicados los testes Chi-cuadrado (en caso de proporciones) o t Student (en caso de medias). Para las análisis de asociación entre (in)seguridad alimentaria y estado nutricional (indicadores antropométricos y bioquímicos) fue usado el teste t Student. Posteriormente se realizó análisis de los efectos ajustados por edad, sexo y renta familiar por persona através de regresión linear múltipla. El teste de Kol- 
mogorov-Smirnov fue usado, previamente, para testar la normalidad de las variables. Todas las análisis fueron realizadas con auxilio del Statistical Package for Social Science (SPSS), versão 12.0.1, considerando como nivel de significado estadístico valores de $p<0,05$.

\section{Resultados}

De la muestra de 282 niños se registró un total de seis recusas y 12 pérdidas (imposibilidad de la colecta de sangre y muestras con hemólisis). Con el objetivo de analizar la posibilidad de sesgo relacionado con la muestra, comparamos la media de edad y la proporción en relación al sexo de los niños de la muestra final (264) con los niños que fueron perdidos. Vimos que las variables no defirieron entre los dos grupos, siendo indicativo de ausencia de sesgo.

El total de 193 niños del presente estudio corresponde a aquellos que tuvieron valores de PCR adecuados (240) y todos sus marcadores bioquímicos evaluados. Al comparar los niños que forman parte de los análisis del presente estudio y los niños que no fueron analizados (con ausencia de informaciones sobre marcadores bioquímicos y/o valores de PCR inadecuados), en relación a parámetros biológicos (sexo, edad), situación de seguridad alimentaria y renta familiar por persona, se pudo notar ausencia de diferencias entre los mismos: proporción de niños de sexo masculino, $\mathrm{p}=0,106$; media de edad (meses), $\mathrm{p}=0,108$; proporción de familias con seguridad alimentaria familiar, $\mathrm{p}=0,429$; proporción de familias con renta familiar por persona $<1 / 2$ salario mínimo; $\mathrm{p}=0,180$.

La EBIA evidenció consistencia interna muy satisfactoria, pues el estadístico Alpha de Cronbach fue de 0,9002. Ese resultado garantiza que, a pesar de entrevistados diferentes de la madre no haber constituido un criterio de exclusión, los mismos tuvieron condiciones de responder la EBIA.

El perfil nutricional de los niños estudiados y la situación de (in)seguridad alimentaria de sus familias está mostrado en la Tabla 1. La evaluación del estado nutricional indicó altas prevalencias de deficiencia de vitamina A, de anemia y de deficiencia de zinc, con valores de 24,4\%, 15,5\% e $15,0 \%$; respectivamente. Relacionado a los indicadores antropométricos revelase la mayor prevalencia de déficit de estatura $(6,2 \%)$ cuando comparado al déficit de peso para la edad (2,1\%). Del total de las familias estudiadas, $35,8 \%$ fueron
Tabla 1. Estado nutricional de niños que frecuentan jardines infantiles, según indicadores antropométricos y marcadores bioquímicos de deficiencias de micronutrientes, y situación de (in)seguridad alimentaria familiar. Paraíba, Brasil, 2008-2009.

\section{Indicadores del estado nutricional y situación} de (in)seguridad alimentaria familiar

n $\%$

Peso/Edad (Escore-z)

Déficit de peso para edad $(<-2)$

Peso adecuado para edad

$89-97,9$

Talla/Edad (Escore-z)

Déficit de estatura $(<-2)$

Estatura adecuada

126,2

Retinol sérico $(\mu \mathrm{mol} / \mathrm{L})$

Deficiencia de vitamina A $(<0,7)$

18193,8

$47 \quad 75,6$

Ausencia de deficiencia de vitamina A

$146 \quad 24,4$

Hemoglobina $(\mathrm{g} / \mathrm{L})$

Anemia $(<110)$

$30 \quad 15,5$

Ausencia de anemia

16384,5

Zinc sérico (ug/dL)

$\begin{array}{lrl}\text { Deficiencia de zinc }(<65) & 29 & 15,0\end{array}$

Ausencia de deficiencia de zinc $\quad 164 \quad 85,0$

Situación de (in)seguridad alimentaria familiar Inseguridad alimentaria grave $\quad 19 \quad 9,8$

Inseguridad alimentaria moderada $\quad 4221,8$

$\begin{array}{lll}\text { Inseguridad alimentaria leve } & 63 & 32,6\end{array}$

$\begin{array}{lrr}\text { Seguridad alimentaria } & 69 & 35,8\end{array}$

Total

193100,0

clasificadas en la categoría de seguridad alimentaria, predominando, por tanto, la condición de inseguridad alimentaria $(64,2 \%)$, siendo más prevalente la forma leve $(32,6 \%)$, seguida por la forma moderada $(21,8 \%) \mathrm{y}$, por último, la forma grave $(9,8 \%)$.

La inseguridad alimentaria cuando asociada a los índices antropométricos (P/E, T/E) y marcadores bioquímicos (retinol sérico, hemoglobina, zinc sérico) del estado nutricional de los niños no mostró asociación estadísticamente significante (Tabla 2).

\section{Discusión}

La aplicación de la EBIA se ha centrado en la determinación de la magnitud de la inseguridad alimentaria familiar, enfocando la vulnerabilidad al problema ${ }^{31}$. Más de la mitad de las familias $(64,2 \%)$ que participaron del estudio estuvieron con inseguridad alimentaria, siendo 32,6\% leve, $21,8 \%$ moderada y $9,8 \%$ grave. Considerando la 
Tabla 2. Medias de Escore-z de peso/edad, Escore-z de talla/edad, retinol sérico, hemoglobina y zinc sérico de niños que frecuentan jardines infantiles, según situación de (in)seguridad alimentaria familiar. Paraíba, Brasil, 2008-2009.

\begin{tabular}{lrrrrrr}
\hline $\begin{array}{l}\text { Índices antropométricos y marcadores } \\
\text { bioquímicos del estado nutricional }\end{array}$ & Media & \pm DP & Media & \pm DP & $\mathbf{p}^{*}$ & $\beta$ \\
\hline Peso/Edad (Escore-z) & 0,4387 & 0,9580 & 0,1564 & 0,9690 & 0,198 & $-0,280$ \\
Talla/Edad (Escore-z) & $-0,2288$ & 1,1425 & $-0,5678$ & 1,1488 & 0,050 & $-0,311$ \\
Retinol sérico ( $\mu$ mol/L) & 0,9188 & 0,3047 & 0,8602 & 0,2587 & 0,159 & $-0,301$ \\
Hemoglobina (g/L) & 11,7754 & 0,8750 & 11,5197 & 1,1126 & 0,281 & $-0,330$ \\
Zinc sérico (ug/dL) & 78,4275 & 14,1313 & 76,0637 & 12,4633 & 0,230 & $-0,238$ \\
\hline
\end{tabular}

" Significancia del Teste $t$ Student. ${ }^{* *}$ Coeficiente de regresión de la regresión linear. Medias ajustadas por edad ( $\geq 24$ meses), sexo (femenino) y renta familiar por persona $\left(<0,25\right.$ salarios mínimos). Coeficiente de determinación $\mathrm{R}^{2}=13,0 \%$. $\mathrm{p}>0,05$.

prevalencia del problema a nivel nacional, se observó, según la Pesquisa Nacional por Amostra de Domicílios de $2009^{32}$, que la inseguridad alimentaria es un problema preocupante, donde 30,2\% de la población estaba en esa situación, siendo considerablemente más prevalente en la región Nordeste (46,1\%). En el Estado de Paraíba, 59,0\% de las familias estaban con seguridad alimentaria y $41,0 \%$ con inseguridad alimentaria, siendo $23,5 \%$ leve, $10,5 \%$ moderada y $7,0 \%$ grave. En estudio realizado en municipios del interior de Paraíba 52,5\% de las familias estaban con inseguridad alimentaria, siendo 23,6\% leve, 17,6\% moderada y $11,3 \%$ grave $^{33}$. Estos resultados confirman las altas prevalencias de inseguridad alimentaria en Brasil, mostrando valores próximos cuando comparados. La vulnerabilidad socioeconómica de la población albo del presente estudio puede explicar las prevalencias de inseguridad alimentaria un poco mayores.

Reflexionando un poco más sobre la población de estudio, cabe resaltar que en el contexto de investigaciones para medir la seguridad alimentaria varias ventajas pueden ser adjudicadas al enfocar niños preescolares. En un ámbito general está claro que, debido a que las repercusiones de los cambios económicos en el estado nutricional de la población no son inmediatas y los individuos tienen diferentes mecanismos de adaptación a las bajas ingestiones de alimentos, los indicadores nutricionales más adecuados para evaluar la seguridad alimentaria son los de poblaciones infantiles de tendencia histórica ${ }^{34}$. Así, también, la condición social y económica es otro aspecto que debe tenerse en vista, pues los grupos de mayor vulnerabilidad a situaciones de inseguridad alimentaria (concepto implícito en la EBIA) son los que figuran en cualquier lista de desposeídos, lo cual cuando asociado al gru- po materno infantil adquiere mayor importan$\mathrm{cia}^{35}$. En el caso de Brasil esos factores pueden ser contemplados al considerar los jardines infantiles como local de estudio. Por lo general estas instituciones están situadas en áreas carentes que abrigan niños de familias de bajos rendimientos económicos y de estratos sociales menos favorecidos. La demanda por estas instituciones es grande, lo cual ha repercutido en un aumento expresivo en la cantidad de locales disponibles y en el número de preescolares beneficiados ${ }^{36-38}$.

La mayor prevalencia de déficit de estatura, cuando comparada con la prevalencia de déficit de peso para la edad, bien como las altas prevalencias de deficiencia de vitamina A, de anemia y de deficiencia de zinc, son resultados de este estudio que coinciden con las tendencias del país: i) el retraso en la talla como característica antropométrica del cuadro epidemiológico del crecimiento infantil que persiste principalmente en grupos vulnerables de la población; ii) la carencia persistente y preocupante de algunos micronutrientes esenciales; iii) la asociación, con los anteriores, del aumento de exceso de peso en diferentes fases de la vida ${ }^{39,40}$.

Si consideramos que la prevalencia de inseguridad alimentaria debería representar las deficiencias nutricionales, el análisis comparativo de los datos muestra un panorama conflictivo. Parece paradoxal que en niños de familias socio-económicamente vulnerables, con alta frecuencia de inseguridad alimentaria, los índices antropométricos muestren prevalencias bien inferiores y su proximidad o superioridad de los valores considerados para definir la normalidad $(95,0 \%)^{22}$.

Considerando el contexto de las deficiencias de micronutrientes, el cuadro epidemiológico se asemeja más al de inseguridad alimentaria en tér- 
minos de frecuencias. Comportamiento similar derivase del conjunto de resultados encontrados en poblaciones escogidas intencionalmente por su vulnerabilidad socioeconómica, estudiando familias constituidas por niños menores de cinco años $^{14-16}$. La importancia del estudio de los micronutrientes vitamina A, hierro y zinc en el contexto de la SAN se relaciona a sus características que los hacen poder clasificar en el grupo de micronutrientes cuya carencia provoca "hambre oculta": i) falta de ubicuidad en la naturaleza (lista de fuentes naturales pequeña); ii) escaso acceso/aceptación, con frecuencia, de las fuentes naturales por uno de los grupos más afectados por la carencia: niños preescolares; iii) consecuencia de las anteriores, escasez o ausencia en la dieta de los grupos vulnerables; $y$ iv) agentes protectores del crecimiento con importantes interacciones metabólicas cuyas deficiencias son problemas de salud pública ${ }^{41}$.

Fueron encontrados cuatro estudios ${ }^{9,13-15}$ abordando la asociación entre índices antropométricos del estado nutricional de niños y la inseguridad alimentaria familiar. En estudio realizado entre familias con niños de 6-18 meses afrocolombianos residentes en Guapi (Colombia) ${ }^{13}$ y en estudio realizado con niños menores de 30 meses en Duque de Caxias, Rio de Janeiro (Brasil $)^{9}$, la asociación entre las variables fue encontrada. Sin embrago, estudios realizados con niños menores de cinco años en São João do Tigre $^{14}$, Pernambuco (Brasil) y Gameleira, Pernambuco (Brasil) ${ }^{15}$ no encontraron asociación estadística entre la inseguridad alimentaria y la desnutrición, coincidiendo con los resultados da la población considerada en este trabajo. En cuanto un estudio ${ }^{9}$ discute la posible capacidad de predicción de la desnutrición con el uso de la EBIA, los estudios en ciudades de Pernambuco ${ }^{14,15}$ sugieren que son factores independientes, por reflejar eventos y representaciones (perspectiva subjetiva de la EBIA) diferentes, a pesar de la complementariedad entre desnutrición e inseguridad alimentaria.

Así, consideramos la necesidad de más estudios sobre la asociación entre la inseguridad alimentaria y el estado nutricional. Estudio reciente sobre la investigación en el tema de SAN en Brasil mostró claramente la inadecuación de las investigaciones considerando los direccionamientos específicos que limitan análisis de la amplitud y complejidad del concepto de $\mathrm{SAN}^{42}$. En ese sentido, hay que destacar que la medición de la SAN por la EBIA puede maximizar el entendimiento de la problemática si otros indicadores son usados en conjunto ${ }^{43}$.

Ninguna asociación estadísticamente significante fue encontrada entre los marcadores bioquímicos del estado nutricional de micronutrientes y la inseguridad alimentaria. Resultados similares para el estado nutricional de vitamina $\mathrm{A}$ y hierro fueron encontrados en poblaciones de las ciudades de Gameleira y São João do Tigre, en Pernambuco, Brasil ${ }^{16}$. Así, siendo, la inseguridad alimentaria evaluada por la EBIA representa una manifestación subjetiva que pode perder su poder discriminatorio de situaciones más específi$\operatorname{cas}^{16}$, como son las deficiencias de micronutrientes. La comprensión de las múltiples interfaces entre la inseguridad alimentaria y el estado nutricional es necesaria para tomar las decisiones adecuadas de políticas públicas.

En ese contexto, considerase que el cuadro epidemiológico brasileño marcado por la coexistencia de enfermedades crónicas no transmisibles, obesidad, carencias de micronutrientes, hambre y desnutrición, entre otros, precisa de acciones integrales que comprendan todo el espectro de la seguridad alimentaria, de la producción al consumo de alimentos ${ }^{44-46}$. Incluso, resultados empíricos han demostrado que la búsqueda por la seguridad alimentaria envuelve el acceso a recursos y medios de producción de alimentos para el autoconsumo ${ }^{47}$. Es conveniente, en ese sentido, resaltar la influencia de la renta familiar como condición básica de la seguridad alimentaria ${ }^{48}$.

\section{Conclusiones}

La población estudiada, caracterizada por el bajo nivel socio-económico e institucionalización, presentó altas prevalencias de inseguridad alimentaria familiar, distantes de las prevalencias dos déficits de estatura y de peso para edad y más próximas de las deficiencias de vitamina $\mathrm{A}$, ferro y zinc. La inseguridad alimentaria no se asoció con los Escore-z de crecimiento ni con las concentraciones de retinol sérico, hemoglobina y zin sérico, resultados que deben ser interpretados cuidadosamente al examinar la consistencia entre la EBIA y los parámetros del estado nutricional de los niños. Investigaciones adicionales en diferentes contextos son necesarias para mayor profundidad sobre el tema. 


\section{Consideraciones finales}

Los resultados del presente estudio pueden ser considerados relevantes en relación al conocimiento científico sobre la medición y análisis de la SAN y para la evaluación de impacto de políticas y programas. Existen considerables estudios sobre el estado nutricional y la frecuencia de enfermedades infecto-contagiosas de niños asistidos en jardines infantiles. Sin embargo, poco se ha estudiado sobre sus especificidades relacionadas con la seguridad alimentaria. La realización de otros estudios posibilitaría entender mejor el contexto de la seguridad alimentaria como prerrequisito para prevenir deficiencias nutricionales. Los resultados del presente estudio sugieren que la EBIA, más que como enfoque epidemioló- gico de la desnutrición, debe ser entendida como abordaje antropológica que expresa adaptaciones ante la escasez de alimentos. Creemos, inclusive, delicado analizar la inseguridad alimentaria como un problema de salud pública, pues es una condición con sus propias especificidades en cuanto a sus factores determinantes y consecuencias. Además, como las deficiencias de macronutrientes representa cada vez menos la realidad del estado nutricional de la población infantil brasileña, coloca-se, tal vez, una nueva conjuntara en la cual la inseguridad alimentaria se encuentra disociada del riesgo de esas deficiencias, tomando parte importante las deficiencias de micronutrientes como sucede, por ejemplo, con la determinación del déficit de estatura por las deficiencias de vitamina A, zinc y hierro.

\section{Colaboradores}

D Figueroa Pedraza participó de la elaboración del proyecto, concepción del artículo, revisión bibliográfica, análisis e interpretación de los datos, redacción, revisión crítica y aprobación final del artículo. D Queiroz participó de la revisión bibliográfica, análisis e interpretación de los datos, redacción, revisión crítica y aprobación final del artículo. AA Paiva participó de la elaboración del proyecto, revisión crítica y aprobación final del artículo. MAL Cunha y ZN Lima participaron de la revisión crítica y aprobación final del artículo. 


\section{Referências}

1. Oliveira JS, Lira PIC, Veras ICL, Maia SR, Lemos MC, Andrade SLS Viana Júnior MJ, Pinto FCL; Leal VS, Batista Filho M. Estado nutricional e insegurança alimentar de adolescentes e adultos em duas localidades de baixo índice de desenvolvimento humano. Rev Nutr 2009; 22(4):453-465.

2. Batista Filho M. O Brasil e a segurança alimentar. Rev Bras Saude Matern Infant 2007; 7(2):121-122.

3. Radimer KL, Olson CM, Greene JC, Campbell CC, Habicht JP. Understanding hunger and developing indicators to assess it in women and children. $J$ Nutr Educ 1992; 24(Supl. 1):36-44.

4. Álvarez MC, Estrada A, Montoya EC, Melgar-Quiñónez H. Validación de escala de la seguridad alimentaria doméstica en Antioquia, Colombia. Salud Publica Mex 2006; 48(6):474-81.

5. Melgar-Quinonez H, Zubieta AC, Valdez E, Whitelaw B, Kaiser L. Validation of an instrument to monitor food insecurity in Sierra de Manantlan, Jalisco. Salud Publica Mex 2005; 47(6):413-422.

6. Melgar-Quinonez HR, Zubieta AC, McNelly B, Nteziyaremye A, Gerardo MF, Dunford C. Household food insecurity and food expenditure in Bolivia, Burkina Faso, and the Philippines. J Nutr 2006; 136(Supl.):S1431-S1437.

7. Pérez-Escamilla R. Experiência internacional com a escala de percepção da insegurança alimentar. Cad Estud Desenv Soc Debate 2005; 2(1):14-27.

8. Segall-Corrêa AM. Insegurança alimentar medida a partir da percepção das pessoas. Estudos Avançados 2007; 21(60):143-154.

9. Pimentel PG, Sichieri R, Salles-Costa R. Insegurança alimentar, condições socioeconômicas e indicadores antropométricos em crianças da Região Metropolitana do Rio de Janeiro/Brasil. $R$ Bras Est Pop 2009; 26(2):283-294.

10. Salles-Costa R, Pereira RA, Vasconcellos MTL, Veiga GV, Marins VMR, Jardim BC, Gomes FS, Sichieri R. Associação entre fatores socioeconômicos e insegurança alimentar: estudo de base populacional na Região Metropolitana do Rio de Janeiro, Brasil. Rev Nutr 2008; 21(Supl):S99-109.

11. Instituto Brasileiro de Geografia e Estatística (IBGE). Pesquisa Nacional por Amostra de Domicílios: Segurança Alimentar 2004. Rio de Janeiro: IBGE; 2006.

12. Alvarado BE, Zunzunegui MV, Delisle H. Validación de escalas de seguridad alimentaria y de apoyo social en una población afro colombiana: aplicación en el estudio de prevalencia del estado nutricional en niños de 6 a 18 meses. Cad Saude Publica $2005 ; 21(3): 724-736$.

13. Fundo das Nações Unidas para a Infância (UNICEF). Estratégia para Melhorar a Nutrição de Crianças e Mulheres nos Países em Desenvolvimento. New York: UNICEF; 1990.

14. Oliveira JS, Lira PIC, Andrade SLLS, Sales AC, Maia SR, Batista Filho M. Insegurança Alimentar e estado nutricional de crianças de São João do Tigre, no semi-árido do Nordeste. Rev Bras Epidemiol 2009; 12(3):413-423.
15. Oliveira JS, Lira PIC, Maia SR, Sequeira LAS, Amorim RCA, Batista Filho M. Insegurança alimentar e estado nutricional de crianças de Gameleira, zona da mata do Nordeste brasileiro. Rev Bras Saude Matern Infant 2010; 10(2):237-245.

16. Oliveira JS, Lira PIC, Osório MM, Sequeira LAS, Costa EC, Gonçalves FCLSP, Batista Filho M. Anemia, hipovitaminose A e insegurança alimentar em crianças de municípios de Baixo Índice de Desenvolvimento Humano do Nordeste do Brasil. Rev Bras Epidemiol 2010; 13(4):651-664.

17. Batista Filho M, Rissin A. A transição nutricional no Brasil: tendências regionais e temporais. Cad Saude Publica 2003; 19(Supl. 1):S181-S191.

18. Brasil. Ministério da Saúde (MS). Conselho Nacional de Saúde. Resolução no. 196 de 10 de outubro de 1996. Diretrizes e Normas Regulamentadoras de Pesquisas Envolvendo Seres Humanos. Diário Oficial da União 1996; 16 out.

19. Brasil. Ministério da Saúde (MS) Pesquisa nacional sobre demografia e saúde da criança e da mulher. Brasil: MS; 2008.

20. Segall Corrêa AM, Pérez-Escamilla R, Maranha LK, Sampaio MFA. Projeto: acompanhamento e avaliação da segurança alimentar de famílias brasileiras: validação de metodologia e de instrumento de coleta de informação. Campinas: Unicamp; 2003. Relatório Técnico.

21. Instituto Internacional de Investigación sobre Políticas Alimentarias (IFPRI). Seguridad Alimentaria en la Práctica: Métodos para Proyectos de Desarrollo Local. Washington: IFPRI; 2003.

22. World Health Organization (WHO). Physical status: the use and interpretation of anthropometry. Geneva: WHO; 1995. (Technical Report Series, V. 854).

23. World Health Organization (WHO). WHO Child Growth Standards: length/height-for-age, weight-forage, weight-for-length, weight-for-height and body mass index-for-age: methods and development. Geneva: WHO; 2006.

24. Furr HC, Tanumiharjo S, Olson JA. Training Manual for assessing vitamin A status by use the modified relative dose response assays. Sponsored by the USAID Vitamin A Field Support. Washington: IVACG; 1992.

25. World Health Organization (WHO). Indicators for assessing vitamin $A$ deficiency and their application in monitoring and evaluating interventions programs. Geneva: WHO; 1996. (Micronutrients series; WHO/ NUT/96.10).

26. World Health Organization (WHO). Iron deficiency anaemia: assessment, prevention and control - a guide for programme managers. Geneva: WHO; 2001. (WHO/NHD/01.3).

27. Sandström B. Diagnosis of zinc deficiency and excess in individuals and populations. Food Nutr Bull 2001; 22(2):133-137.

28. Hess SY, Peerson JM, King JC, Brown KH. Use of serum zinc concentration as an indicator of population zinc status. Food Nutr Bull 2007; 28(Supl. 3):S403-S429. 
29. Thurnham DI, Mccabe GP, Northrop-Clewes CA, Nestel P. Effects of subclinical infection on plasma retinol concentrations and assessment of prevalence of vitamin A deficiency: meta-analysis. Lancet 2003; 362(9401):2052-2058.

30. Oviedo HC, Campo-Arias A. Aproximación al uso del coeficiente alfa de Cronbach. Rev Col Psiquiatria 2005; 34(4):572-580.

31. Melgar-Quinonez H, Hackett M. Measuring household food security: the global experience. Rev Nutr 2008; 21(Suppl):S27-S37.

32. Instituto Brasileiro de Geografia e Estatística (IBGE). Pesquisa Nacional por Amostra de Domicílios: Segurança Alimentar 2004/2009. Rio de Janeiro: IBGE; 2010.

33. Vianna RPT, Segall-Corrêa AM. Insegurança alimentar das famílias residentes em municípios do interior do estado da Paraíba, Brasil. Rev Nutr 2008; 21(Supl):S111-S122.

34. Figueroa Pedraza D. Medición de la Seguridad Alimentaria y Nutricional. RESPYN 2005; 6(2). [periódico na Internet]. [acessado 2010 abr 28]. Disponível em: http://www.uanl.mx/publicaciones/respyn/ vi/2/ensayos/MedicionSAyN.htm

35. Figueroa Pedraza D. Grupos vulnerables y su caracterización como criterio de discriminación de la seguridad alimentaria y nutricional en Brasil. Rev Bras Saude Matern Infant 2005; 5(3):367-375.

36. Silva MV, Ometto AMH, Furtuoso MCO, Pipitone MAP, Sturion GL. Acesso à creche e estado nutricional das crianças brasileiras: diferenças regionais, por faixa etária e classe de renda. Rev Nutr 2000; 13(3):193-199.

37. Bueno MB, Marchioni DML, Fisberg RM. Evolução nutricional de crianças atendidas em creches públicas no Município de São Paulo, Brasil. Rev Panam Salud Publica 2003; 14(3):165-170.

38. Zöllner CC, Fisberg RM. Estado nutricional e sua relação com fatores biológicos, sociais e demográficos de crianças assistidas por creches da Prefeitura do Município de São Paulo. Rev Bras Saude Matern Infant 2006; 6(3):319-328.
39. Brasil. Ministério da Saúde (MS) Política Nacional de Alimentação e Nutrição. 1a Edição. Brasília: MS; 2012. (Série B. Textos Básicos de Saúde).

40. Coutinho JG, Gentil PC, Toral N. A desnutrição e obesidade no Brasil: o enfrentamento com base na agenda única da nutrição. Cad Saude Publica 2008; 24(Supl. 2):S332-S340.

41. Singh M. Role of micronutrients for physical growth and mental development. Indian J Pediatr 2004; 71(1):59-62.

42. Prado SD, Gugelmin SA, Mattos RA, Silva JK, Olivares PSG. A pesquisa sobre segurança alimentar e nutricional no Brasil de 2000 a 2005: tendências e desafios. Cien Saude Colet 2010; 15(1):7-18.

43. Kepple AW, Segall-Corrêa AM. Conceituando e medindo segurança alimentar e nutricional. Cien Saude Colet 2011; 16(1):187-199.

44. Burlandy L. Segurança alimentar e nutricional e saúde pública. Cad Saude Publica 2008; 24(7):14641465.

45. Burlandy L. Transferência condicionada de renda e segurança alimentar e nutricional. Cien Saude Colet 2007; 12(6):1441-1451.

46. Fonseca AMM, Viana ALA. Direito à saúde, atenção básica e transferências condicionadas de renda na América Latina. Cien Saude Colet 2007; 12(6):15051512.

47. Lang RMF, Almeida CCB, Taddei JAAC. Segurança alimentar e nutricional de crianças menores de dois anos de famílias de trabalhadores rurais Sem Terra. Cien Saude Colet 2011; 16(7):3111-3118.

48. Segall-Corrêa AM, Marin-Leon L, Helito H, PérezEscamilla R, Santos LMP, Paes-Sousa R. Transferência de renda e segurança alimentar no Brasil: análise dos dados nacionais. Rev Nutr 2008; 21(Supl.): S39-S51.

Artigo apresentado em 29/11/2012

Aprovado em 17/12/2012

Versão final apresentada em 18/12/2012 